\title{
A Psychosocial Support Website From the British Association of Dermatologists for People Living With a Skin Condition: Mixed Methods Evaluation
}

Connor Heapy $^{1 *}$, BSc, MSc, PhD; Kerry Montgomery ${ }^{1 *}$, BSc, MSc, PhD; Steven Ersser ${ }^{2}$, BSc (Hons), RGN, PhD, PFHEA; Matt Gass ${ }^{3}$, BA (Hons); Nina Goad ${ }^{3}$, BA (Hons); Andrew R Thompson ${ }^{4}$, BA (Hons), DClinPsy

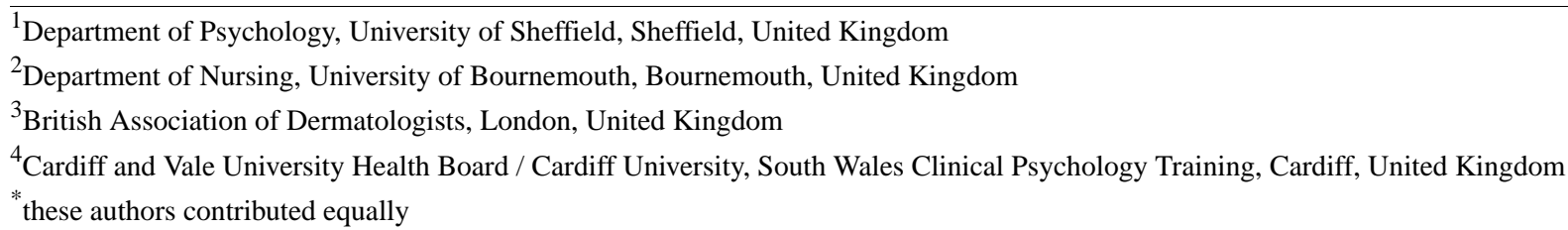

\section{Corresponding Author:}

Andrew R Thompson, BA (Hons), DClinPsy

Cardiff and Vale University Health Board / Cardiff University

South Wales Clinical Psychology Training

11th Floor, Tower Building

70 Park Place

Cardiff, CF10 3AT

United Kingdom

Phone: 442920870582

Email: thompsona18@ cardiff.ac.uk

\section{Abstract}

Background: There is a lack of psychological support for individuals with skin conditions, and few low-intensity self-help interventions are available.

Objective: This study aimed to test the acceptability and usability of a support website and its embedded self-help resources.

Methods: A mixed methods approach was utilized. A total of 583 participants (426 with a skin condition, 97 relatives and friends, and 60 dermatology professionals) viewed the British Association of Dermatologist's SkinSupport website and then completed a survey about their well-being and the usability of the website. A comparison group comprising 816 participants also completed the well-being measures. In total, 37 participants (19 living with a skin condition, and the relatives and friends of individuals with a skin condition, and 18 dermatology professionals) viewed the SkinSupport website and then took part in focus groups. Participants were recruited via social media, professional networks, and volunteer lists. Data from the survey were analyzed using descriptive and inferential statistics and qualitative content analysis.

Results: Both quantitative and qualitative responses suggest that the SkinSupport website was viewed positively by both patients and health professionals. Overall, 79.8\% (417/523) of individuals with a skin condition, and the relatives and friends of individuals with a skin condition, said that they would use the website again; and 86.7\% (52/60) of dermatology professionals said that they would recommend the site to somebody with a skin condition. Qualitative responses related to the website fell into 4 key themes: (1) appearance, (2) use and navigation, (3) information, and (4) areas for development.

Conclusions: The SkinSupport website was considered acceptable and usable. A range of areas requiring modification were identified. The website provides a useful resource that patients can access freely. Given the lack of services available to patients with skin conditions, health care professionals could routinely inform patients of this resource at assessment.

(JMIR Dermatol 2020;3(1):e17052) doi: 10.2196/17052

\section{KEYWORDS}

dermatology; psychology; surveys and questionnaires; interview; mobile phone 


\section{Introduction}

\section{Background}

Skin conditions are common, affecting $54 \%$ of the UK population in any 12-month period [1]. Approximately 13 million people in England and Wales visit their general practitioner (GP) with a skin complaint each year, making it the most frequent reason for patients to visit their GP with a new problem $[1,2]$. Skin conditions may lead to difficulties in emotional, psychological, and social functioning [1,3]. Despite the potential negative impact that skin conditions can have on psychological well-being, access to psychological support for dermatology patients is acknowledged to be limited $[1,4]$. Although support websites and interventions exist for the group more broadly described as having a visible difference, many individuals with a skin condition will not identify themselves as having a visible difference and may, therefore, not find, or feel targeted by, such resources. In addition, there are many nonappearance-based issues associated with having a skin condition that do not apply to many other conditions that lead to a visible difference, such as pain and itch. As a result, the All Parliamentary Group on Skin has identified the provision of psychological services for people with skin conditions as a priority area for attention in 2019 [5].

Web-based self-help support has the potential to offer a cost-efficient method of addressing some of the gaps in the provision of psychosocial support for dermatology patients [5]. Such support can be considered as a low-intensity psychological intervention and is well-suited to individuals experiencing low-to-moderate levels of psychological distress [6]. Individuals could access such support in place of, or before, receiving more intensive forms of psychological therapy or support. Indeed, web-based self-help support has been found to improve individual well-being with a range of chronic health conditions [7]. However, there are few web-based self-help options available for people with skin conditions, and those that are available are condition-specific and not widely accessible (eg, cognitive-behavioral therapy-based web support for psoriasis [8]). Therefore, there is a need for a widely available self-help support tool for individuals with a range of skin conditions.

To address this need for psychological resources for individuals with a skin condition, the British Association of Dermatologists (BAD) launched a website, called SkinSupport [9], in July 2015. Skin Support was designed to support people living with a range of skin conditions and their relatives and friends, and as a signposting tool for professionals. The website was developed by a range of health care professionals and patient representatives. It includes access to downloadable self-help resources that have been subject to some evaluation. SkinSupport hosts a range of skin-specific psychosocial self-help information aimed at helping people to address common problems that are sometimes associated with skin conditions, such as how to regain confidence and improve low mood [10,11]. It also contains information and resources on different skin conditions, including treatment and causes.

\section{Objectives}

This study aimed to examine the acceptability and usability of the BAD SkinSupport website for people living with skin conditions and their relatives and friends. In addition, the acceptability and usability of the website as a signposting resource for professionals working with people living with skin conditions was examined. Evaluating this web-based resource is vital in ensuring that it serves its intended purpose of providing low-intensity psychosocial support for dermatology patients.

\section{Methods}

\section{Design}

A mixed methods approach was used to collect both qualitative and quantitative data using a web-based survey and focus group discussions. Qualitative methods using focus groups were adopted to capture novel, unanticipated responses that may be missed by a predetermined questionnaire [12]. Quantitative questionnaire survey methods were adopted to help summarize key acceptability and usability information across a large sample of individuals, allowing the generalizability of the findings to be examined. Using a single method approach to this question, therefore, would mean that crucial information on the acceptability and usability of the website would be missed. This pragmatic, mixed methods approach, therefore, allowed for a comprehensive assessment of the acceptability and usability of the SkinSupport website $[13,14]$.

\section{Participants}

Both the questionnaire survey and the focus group were open to (1) individuals with a skin condition, (2) the relatives and friends of someone with a skin condition, and (3) dermatology professionals. To be involved in the study, participants were required to be able to speak fluent English and be aged $\geq 16$ years. Any professional working with people with skin conditions was eligible to participate (eg, dermatologists, nurses, GPs, mental health workers and charities, etc).

Participants for the questionnaire survey (evaluation group) were recruited using a convenience sampling method. Participants for the focus groups were recruited via a purposive sampling method. For both parts of the study, participants living with a skin condition and their relatives and friends were recruited via a number of sources, including social media and volunteer lists (university and charity lists). Dermatology professionals were recruited via professional dermatology bodies, dermatology special interest groups, and staff working at a local National Health Service well-being service.

A comparison group of participants was also recruited for this study. These were individuals who had chosen to visit the SkinSupport website for purposes unrelated to the research project and then followed a link to a questionnaire on the website, inviting them to take part. This sample was recruited to check whether the participants who were involved in the website evaluation were typical of those who visited the SkinSupport website. Variables relevant to individuals with skin conditions were recorded and compared between the 2 samples. 


\section{Procedure}

For the survey, participants in the evaluation group followed a web-based link to the questionnaire on the web-based survey platform Qualtrics. The survey first instructed participants to follow a link to the SkinSupport website before answering related questions. Participants in the comparison group were individuals who had visited the SkinSupport website before the current evaluation started. These participants were also asked to complete a series of established, validated questionnaires (patient health questionnaire 2 [PHQ-2], generalized anxiety disorder questionnaire 2 [GAD-2], and dermatology quality of life index [DLQI]).

In total, 3 focus groups were conducted for dermatology professionals and 3 focus groups for people living with skin conditions, and the relatives and friends of individuals with a skin condition. Participants were first sent a link to the website and asked to look at it. In addition, the website was displayed during the focus group discussion and participants were asked to comment on particular aspects. Focus groups were run by 2 authors of this paper, separately: $\mathrm{CH}$ and KM. Some focus group discussions took place in university settings and others in hotel conference room settings. A semistructured interview schedule was used to guide the focus groups, and the discussions were recorded using an encrypted digital recorder. The focus groups lasted approximately $60 \mathrm{~min}$. Data were transcribed and uploaded into NVivo version 11 (QSR International) for thematic analysis. The qualitative data analysis was conducted by KM with supervision provided by AT.

Ethical approval was obtained from the Department of Psychology Ethics Committee, University of Sheffield.

\section{Materials}

The web-based survey assessed the acceptability and usability of the website as well as relevant demographic and clinical information. The acceptability and usability of the website were assessed by asking participants in the evaluation group for their opinions on a range of aspects of the website, including: (1) the appeal, (2) ease of use, (3) quality of information provided, (4) quality of self-help information, and (5) whether they would recommend the site.

The appeal of the website was assessed by asking participants to rate the home page and the rest of the website on a scale from $O$ (not appealing) to 100 (very appealing). Participants were also asked to assess the balance of pictures and text on a 4-point Likert scale from poor to excellent. Ease of use was assessed by asking participants to rate on a 4-point Likert scale how easy they found the website to use, from very easy to use to very difficult to use. Quality of information provided was assessed by asking participants how helpful they found the information on the website from 0 (very unhelpful) to 100 (very helpful). Quality of self-help information provided was assessed by asking participants to rate on a 4-point Likert scale how easy the information was to understand, from clear and easy to understand to very difficult to understand. Participants were also asked to rate how helpful the self-help resource was from $O$ (very unhelpful) to 100 (very helpful) and if they would use the techniques described in the self-help information from 0 (very unlikely) to 100 (very likely). Participants were also asked to rate on a 5-point Likert scale from definitely to definitely not whether they would use the website again, whether they would recommend the website to people living with a skin condition, and whether they would recommend the website to people supporting someone with a skin condition. Participants were also asked to provide any further comments on each of these areas in free-text response boxes.

The following demographic information was collected: age, gender, country of residence, ethnic group, and diagnosed skin condition (if relevant).

The DLQI is a 10-item measure of the impact of the skin condition on the patient's life in the last week. The DLQI has demonstrated good validity, reliability, and responsiveness to changes in a range of skin conditions $[15,16]$.

PHQ-2 [17] is a 2-item measure of depression. PHQ-2 demonstrated good criterion and construct validity. PHQ-2 has a maximum score of 6 , and a score of $>3$ is indicative of symptoms of major depression, with a sensitivity of $83 \%$ and specificity of $90 \%$ [17].

GAD-2 is a 2-item measure of the symptoms of GAD. GAD-2 has a maximum score of 6 , and a score of $>3$ is indicative of clinically significant symptoms of GAD [18]. GAD-2 showed good sensitivity for GAD $(88 \%)$, panic disorder $(76 \%)$, and social anxiety disorder (70\%) and good specificity (81\%-83\%) for all 3 disorders [17].

\section{Website Development}

The website development was guided from the outset by an expert advisory panel, comprising patients, psychiatrists, psychologists, dermatologists, and communications professionals. This panel was able to help guide the BAD through the many complexities involved in providing advice to potentially vulnerable service users or those in acute distress, and, crucially, to collate, evaluate, and commission the profusion of materials required. A large proportion of time and resources were allocated to the development and testing of the website, before a national rollout. This included beta testing of the site with medical professionals, the public, and patients. Patient information materials on the website were reviewed by a medical committee and seconded by experts every 3 years. This review date can be brought forward in response to known changes, such as withdrawal of a named drug or updates to guidelines.

\section{Analysis}

For the survey data, descriptive statistics were used to provide information on the demographics of participants in the evaluation group completing the survey. The current survey data for people living with skin conditions were compared with data collected by the BAD, before the launch of the survey $(\mathrm{n}=816)$, to examine any differences in anxiety, depression, and quality of life between website visitors and survey participants.

Qualitative free-text responses from the survey were analyzed using qualitative content analysis (QCA) [19]. QCA is a systematic text analysis technique that preserves the advantages of quantitative analysis, allowing frequencies of data to be 
reported while also providing a method to examine the experiences of participants using the SkinSupport website.

Qualitative data collected from the focus groups were analyzed using thematic analysis [20]. Thematic analysis is a method used to "identify, analyse and report patterns within data" to explain a particular phenomenon of interest [20]. Analysis of the focus group transcripts began by using line-by-line analysis, from which a list of key themes was generated from each group. These initial themes were then compared and contrasted to identify how the themes from each group fitted together. The aim was to preserve the integrity of the feedback gained from the individual groups within the final set of themes and to achieve internal saturation [21]. Detailed records of the analysis containing excerpts from the transcripts were maintained to enable checking.

Demographic data (age, gender, and ethnicity) of individuals in the evaluation group who would recommend the website to others were compared with those who would not recommend the website to others using a $t$ test and chi-square test of homogeneity. Ethnicity was recoded into a dichotomous variable, to white and nonwhite, due to the relatively small number of participants with many of the nonwhite ethnic categories.

The qualitative results from the survey and focus groups were analyzed separately. These findings were then combined and summarized for the purpose of this study.

\section{Results}

\section{Participant Characteristics}

In total, 816 participants completed the web-based survey as part of the comparison group (no demographic information was collected) and 583 participants completed the web-based survey as part of the evaluation group (426 participants living with a skin condition, 97 relatives and friends of individuals living with skin conditions, and 60 dermatology professionals). The participants living with a skin condition, and the relatives and friends of individuals with a skin condition, were predominately white $(394 / 463,85.1 \%)$, female $(407 / 477,85.3 \%)$, living in the United Kingdom (465/473, 98.3\%), and aged between 16 and 74 years (mean age 38.6, SD 12.6 years). The most common skin conditions reported were eczema (132/396, 33.3\%), psoriasis $(61 / 396,15.4 \%)$, and acne $(43 / 396,10.9 \%)$, with some participants reporting more than one skin condition (Table 1). Health professionals included dermatologists (26/60, 43.3\%), dermatology nurses $(16 / 60,26.7 \%)$, psychological practitioners $(6 / 60,10.0 \%)$, GPs and general practice nurses $(3 / 60,5.0 \%)$, and charity workers $(2 / 60,3.4 \%)$.

In addition, 37 participants took part in the focus groups (19 participants living with a skin condition, and the relatives and friends of people living with a skin condition, across the 3 focus groups, and 18 dermatology professionals across the 3 focus groups).

\section{Psychological Well-Being}

Of those participants in the evaluation group, 33.9\% (132/389) reported clinically significant symptoms of depression. In addition, $37.0 \%(144 / 389)$ of the participants in the evaluation group reported clinically significant symptoms of anxiety. Quality of life related to the skin condition was examined in those with a skin condition in the evaluation group (those without a skin condition were not assessed on quality of life), and $88.7 \%(345 / 389)$ of participants indicated that their skin condition had an effect on their quality of life, ranging from a small to an extremely large effect (Table 2).

A two-tailed independent $t$ test found that participants in the comparison group scored significantly higher in depression (PHQ-2: mean 2.77, SD 1.91) than participants in the evaluation group (mean 2.23 , SD $1.89 ; \mathrm{t}_{1203}=-4.67 ; P<.001$ ). In addition, participants in the comparison group scored significantly higher in anxiety (GAD-2: mean 2.75, SD 1.97) than those in the evaluation group (mean 2.38, $\mathrm{SD} 1.99 ; \mathrm{t}_{1203}=-3.05 ; P=.002$ ). Finally, participants in the comparison group reported poorer quality of life related to their skin condition (DLQI: mean 13.16, SD 7.84) than participants in the evaluation group (mean 9.53, SD 7.48; $\left.\mathrm{t}_{1207}=-7.63 ; P<.001\right)$. 
Table 1. Sample characteristics from individuals with a skin condition, and the relatives and friends of individuals with a skin condition, completing the web-based survey $(\mathrm{N}=523)$.

\begin{tabular}{|c|c|}
\hline Sample characteristic & Values, n (\%) \\
\hline \multicolumn{2}{|l|}{ Skin condition ${ }^{\mathrm{a}}$} \\
\hline Eczema, dermatitis, and prurigo & $132(25.2)$ \\
\hline Acne & $43(8.2)$ \\
\hline Psoriasis & $61(11.6)$ \\
\hline Undiagnosed skin problems (dry, oily, itchy, or flaky skin) & $31(5.9)$ \\
\hline Rosacea & $24(4.5)$ \\
\hline Alopecia & $13(2.4)$ \\
\hline Vitiligo & $12(2.3)$ \\
\hline Scarring & $12(2.3)$ \\
\hline Other & $68(13.0)$ \\
\hline Total that provided skin condition information & $396(75.7)$ \\
\hline \multicolumn{2}{|l|}{ Gender } \\
\hline Male & $65(12.4)$ \\
\hline Female & $407(77.8)$ \\
\hline Other & $5(1.0)$ \\
\hline Total that provided gender information & $477(91.2)$ \\
\hline \multicolumn{2}{|l|}{ Ethnicity } \\
\hline White & $394(75.3)$ \\
\hline Asian or Asian British & $39(7.4)$ \\
\hline Black or African or Caribbean or Black British Caribbean & $9(1.7)$ \\
\hline Other & $8(1.5)$ \\
\hline Preferred not to say & $13(2.4)$ \\
\hline Total that provided ethnicity information & $463(88.5)$ \\
\hline
\end{tabular}

${ }^{\mathrm{a}}$ These data are from participants with a skin condition and not relatives and friends of individuals with a skin condition.

Table 2. Dermatology quality of life scores from participants with a skin condition who completed the web-based survey (N=389).

\begin{tabular}{ll}
\hline Impact of skin condition on quality of life & Values, $\mathrm{n}(\%)$ \\
\hline No effect & $44(11.3)$ \\
Small effect & $91(23.4)$ \\
Moderate effect & $110(28.3)$ \\
Very large effect & $104(26.7)$ \\
Extremely large effect & $40(10.3)$ \\
\hline
\end{tabular}

The following information presented refers only to participants from the evaluation group, as only data on depression, anxiety, and quality of life were collected from the comparison group.

\section{Appearance of the Website}

\section{Descriptive Statistics}

Participants were asked to rate the appearance of the SkinSupport homepage and other pages on a scale of 0 (not appealing) to 100 (very appealing). Participants with a skin condition, and the relatives and friends of individuals with a skin condition, positively rated the appearance of the homepage (mean 74.92 ,
SD 17.86) and the rest of the website (mean 71.49, SD 20.30). Similarly, professionals positively rated the appearance of the homepage (mean 77.32, SD 17.11) and the rest of the website (mean 76.48, SD 17.05).

The balance of pictures and text was rated on a 4-point Likert scale, ranging from poor to excellent. The majority of participants with a skin condition, and the relatives and friends of individuals with a skin condition, rated the balance of pictures and text as excellent $(183 / 569,32.2 \%)$ or good (309/569, $54.3 \%$ ). Similarly, the majority of professionals rated the balance 
of text and images as excellent $(18 / 58,31 \%)$ or good $(29 / 58$, $50 \%)$.

\section{Views of Individuals With Skin Conditions and the Relatives and Friends of Individuals With a Skin Condition}

The appearance, layout, and images used on the website were generally viewed positively by those with a skin condition, and the relatives and friends of someone with a skin condition (Multimedia Appendix 1). The website was described as looking professional and visually appealing. The images of patients being used on the website were viewed positively, although some commented that the images were not diverse enough and omitted younger age groups and ethnic minorities. In addition, some people suggested that more pictures of the different skin conditions should be added. There were conflicting views of the balance of text and image, with some saying the balance was good and others commenting that the website was too text heavy. Although some participants described the colors positively (eg, as calming), others described the colors as bland and the words as not being distinct enough, potentially affecting readability.

\section{Views of Dermatology Professionals}

In general, professionals viewed the website positively, describing it as professional and visually appealing (Multimedia Appendix 1). As with the patient group, there were conflicting views regarding the images used on the website and the balance of images and text. Some participants commented that there was a lack of images in general, and more specifically, a lack of images representing ethnic minorities. Others commented that they liked the use of images of real patients. Participants suggested that adding videos to describe people's experiences could be helpful. One professional suggested that the medical information on the website was not accurate, and links to other websites needed updating. Finally, 1 dermatologist suggested that a separate area on the website for children and young people would be helpful, as the current website may not be appealing to a younger age group.

\section{Use and Navigation}

\section{Descriptive Statistics}

Participants were asked to rate the difficulty in understanding the information displayed on the website, and the language and terminology used on the website, on a scale from 0 (very difficult to understand) to 100 (very easy to understand). Those with a skin condition, and the relatives and friends of individuals with a skin condition, rated the information displayed on the website (mean 78.04, SD 20.15) and the language and terminology used (mean 77.49, SD 20.94) as easy to understand. Dermatology professionals also rated the information displayed on the website (mean 79.15, SD 20.23) and the language and terminology used (mean 77.90, SD 22.25) as easy to understand. In addition, $96.6 \%(504 / 522)$ of participants with a skin condition, and the relatives and friends of individuals with a skin condition, and $96.6 \%(56 / 58)$ of dermatology professionals, reported that the website was easy, or very easy to use.

\section{Views of Individuals With Skin Conditions and the Relatives and Friends of Individuals With a Skin Condition}

Participants reported finding the website easy to use and navigate through, even on a smartphone (Multimedia Appendix 1). However, some participants said that it was difficult to locate materials on the website and that the structure could be improved, as it was difficult to navigate to previous pages. Similarly, 1 participant commented that the navigation bar was inconsistent throughout the website and should be made consistent. Some participants also suggested that a search function should be added to each page. In addition, it was suggested that the search function should be made more intelligent so that it could predict what condition you were looking for, even if there was imprecision in the typed search term. Finally, some participants commented on the difficulty of navigating through the $A-Z$ list of conditions.

\section{Views of Dermatology Professionals}

Professionals generally thought that the website was user friendly and easy to navigate (Multimedia Appendix 1). Although 1 participant felt that the use of medical jargon was avoided on the website, other participants reported that the patient information leaflets were written in unfriendly language, and some of the leaflets were too complex. It was suggested that a button that quickly navigates participants back to the home page would be useful. Finally, 1 participant was concerned about links on the website that took participants to information that did not inform or reassure sufficiently.

\section{Information on the Website}

\section{Descriptive Statistics}

Participants rated how helpful the information on the website was on a scale from 0 (not helpful) to 100 (very helpful). Participants with a skin condition had a mean score of 77.27 (SD 18.50), and the relatives and friends of someone with a skin condition had a mean score of 78.53 (SD 19.64), suggesting that they found the information helpful. In addition, $97.3 \%$ (468/481) of participants with a skin condition and the relatives and friends of someone with a skin condition and $100 \%$ (37/37) of dermatology professionals rated the self-help information on the website as clear and easy to understand, or somewhat easy to understand.

\section{Views of Individuals With Skin Conditions and the Relatives and Friends of Individuals With a Skin Condition}

Participants generally reported that the website was comprehensive and helpful (Multimedia Appendix 1). However, some commented on the fact that some information was too long and wordy. Some participants noticed missing information, for example, on different subtypes of certain skin conditions. Others commented on the fact that they learned no new information from the website. It was also suggested that the purpose of the website should be clarified. Some commented that the information should be made less medical; in particular, the use of the term psychodermatology should be removed. Finally, some participants said that the information was too 
heavily focused on adults, with too much focus on psoriasis specifically.

\section{Views of Dermatology Professionals}

The information on the website received mixed comments from dermatology professionals. Some thought the website needed more information on eczema (Multimedia Appendix 1). Others said that there was too much information on certain topics and that this may be off putting for some patients. One professional suggested that the use of videos to help with mindfulness exercises would be useful. In addition, some professionals suggested that the language of the website was overly medical in places and might benefit from revision. On certain topics, the information was said to be inaccurate. Furthermore, some professionals commented that the material was too focused on adults with a greater focus on psoriasis. Finally, 2 professionals reported being disappointed with the quality of information contained in some third-party materials on the website, including inaccurate crisis line information.

\section{Areas for Development}

\section{Descriptive Statistics}

Individuals with a skin condition, and the relatives and friends of individuals with a skin condition, were asked whether they would visit the website again; $42.3 \%$ (221/523) reported that they definitely would and $37.5 \%(196 / 523)$ reported that they probably would. In terms of recommending the website, $48.9 \%$ (256/523) said that they definitely would and 35.0\% (183/523) said they probably would recommend the website to others living with a skin condition.

Dermatology professionals were asked whether they would recommend the website to people living with a skin condition; $75 \%(45 / 60)$ said that they definitely would, and $12 \%(7 / 60)$ said that they probably would. In terms of recommending the website to individuals supporting others with a skin condition, $65 \%(39 / 60)$ of dermatology professionals reported that they definitely would, and $18 \%$ (11/60) reported that they probably would.

Demographic data (ie, age, gender, and ethnicity) were compared between those individuals with a skin condition, and the relatives and friends of individuals with a skin condition, who would recommend (definitely would or probably would) the website to others and those who would not. There was no difference in age between those who would recommend the website to others (mean 38.7, SD 12.67) and those who would not recommend the website to others (mean 38.0, SD 12.45; $\left.\mathrm{t}_{473}=4.56 ; P=.65\right)$. In addition, no differences were found in gender; $85.6 \%$ (338/395) women would recommend the website compared with $90 \%$ (69/77) women who would not recommend the website, a nonstatistically significant difference in proportions of $0.04(P=.35)$. Similarly, no differences were found in ethnicity; $84.1 \%$ (332/395) white participants would recommend the website compared with $64.9 \%$ (61/94) white participants who would not recommend the website, a nonstatistically significant difference in proportions of 0.19 $(P=.21)$.

\section{Views of Individuals With Skin Conditions and the Relatives and Friends of Individuals With a Skin Condition}

Participants were asked if there were areas of SkinSupport that could be developed. A total of 2 key areas were highlighted: (1) case studies and stories of people living with skin conditions, and (2) addressing the impact of stigma on people living with skin conditions (Multimedia Appendix 1). Some in 2 of the focus groups felt that the stigma surrounding skin conditions needed to be acknowledged on SkinSupport and could be added as another support category. Participants also thought it beneficial to have information about how skin is portrayed in the media, the view of skin conditions as contagious, and what the $\mathrm{BAD}$ is doing to challenge the myths around skin conditions.

\section{Views of Dermatology Professionals}

Professionals outlined several key areas that could be developed on the SkinSupport website. Regarding content, participants reported that information on habit reversal would be useful, and a question and answer section (Multimedia Appendix 1). One participant suggested that providing further information on common problems such as relationship issues and sleep would be beneficial, while also highlighting the connection between physical and mental health. Participants discussed having more condition-specific self-help available would be useful as a lot of the current information relates to psoriasis. Information on social stigma was also mentioned as being beneficial for patients. Finally, participants felt that the website needed further development to be applicable to young people.

\section{Discussion}

\section{Principal Findings}

This mixed methods study provides a detailed evaluation of the acceptability and usability of a psychosocial web-based support resource: SkinSupport. Participants included dermatology patients, and the relatives and friends of individuals with a skin condition, and professionals who work with individuals with skin conditions. Overall, SkinSupport was evaluated positively, and as such has the potential to be a useful resource for people living with skin conditions. For the quantitative measures of the website, the majority of participants gave positive ratings of the website's appearance, use, and navigation, and included information. In addition, participants provided positive feedback on the colors used on the website, the balance of pictures and text, and viewed the information provided as comprehensive for their condition. These quantitative results were generally supported by qualitative responses from participants in the questionnaire response boxes and in the focus groups.

Although the website was generally evaluated positively, several areas for improvement were identified. More specifically, recommendations relating to the appearance of the website include, adding more diverse images (ie, ethnic minorities, other age groups), adding more pictures of the conditions themselves, replacing text heavy sections with images, considering the use of videos, and adding further experiential accounts or stories. 
Recommendations relating to the use and navigation of the website include adding a home button to each page to improve navigation, adding a search function to each page to improve navigation, improving the search function so that it is more general and intelligent (ie, specific words do not need to be typed in to find particular resources), and developing a separate area on the website for children and young people.

Recommendations relating to the information contained on the website include checking the accuracy of all the medical information on the website (including third-party links), reducing medical or unfriendly language (eg, the use of the word psychodermatology), making sure the information is more balanced and less focused on psoriasis, adding information that addresses the impact of stigma, relationship issues, and better signposting of the information on sleep, and adding a question and answer section to the website.

Some caution should be taken when generalizing the findings of the survey in this study to all users of the SkinSupport website. Participants who were involved in the evaluation of the website in this study demonstrated fewer symptoms of depression and anxiety and higher dermatology-related quality of life than participants in the comparison sample drawn from the existing visitors. This finding suggests that those who provided feedback on the survey may not be representative of those who typically visit the website. However, although there are some areas of the website where it could be plausible that individuals who are more distressed may evaluate the site differently (eg, self-help materials), for most areas (eg, appearance, use, and navigation), this seems unlikely. It is important to note that even those participants in the evaluation group of the website scored highly in depression (mean 2.23 SD 1.89 from a maximum of 6 , the cutoff for major depression is $3 ; 132 / 389,33.9 \%$ reached clinically significant levels of depression) and anxiety (mean 2.38 SD 1.99 from a maximum of 6 , the cutoff for generalized anxiety is $3 ; 144 / 389,37.0 \%$ reached clinically significant levels of anxiety) supporting the suggestion that such a self-help resource is needed for this population. Asking patients if they have visited this website may provide a useful rough guide for clinicians in identifying those individuals that require further psychological support.

It is important to note that 2 different constructs were being measured in this study: perceived usefulness of the website (ie, views from patients) and perceived usefulness of the website by others (ie, views from relatives, friends, and health care professionals). In general, the views of all groups aligned regarding each aspect of the website, meaning we can be confident in our conclusions on the strengths and weaknesses of the website in its current form. Indeed, many comments were repeated across groups (eg, images not diverse enough). Understandably, health care professionals were more likely to comment on the accuracy of the information and to suggest the addition of specific information (eg, on habit reversal). Overall, the website was perceived as useful (by patients) and perceived as useful by others (by relatives, friends, and health care professionals).

\section{Limitations}

A methodological limitation of this study is that participants may not have reviewed the entire website before completing the survey or being involved in the focus groups. The website contains a lot of content, and it would take a significant amount of time to review it all. As a result, participants may have been commenting only on small sections of the website that they had reviewed. We cannot comment on the areas of the website that participants visited, or the time they spent on the website, as these data were not collected. Although all visitors to the website were invited to complete questionnaires (to form the comparison group), it is not possible to comment on the overall response rate of those who visited the website as we did not collect this data. In addition, demographic data were not collected from this comparison sample to reduce the burden and increase the response rate. As a result, the comparison group may not be representative of those who visit the website. Despite these limitations, the study involved a large sample size and captured a wide range of views from patients, relatives, and friends of individuals with a skin condition, and health care professionals. The mixed methods approach meant that comprehensive responses were collected using different types of data regarding many areas of the SkinSupport website.

A further limitation of the study is that some of the authors of the paper (MG, AT, and NG) were also on the advisory panel for development of the website, increasing the risk of bias. However, neither the quantitative nor qualitative data were analyzed by these authors to reduce the likelihood of bias and increase the rigor of the analysis.

Future studies are needed to investigate the effectiveness of some of the untested self-help materials contained on the website, and the tested interventions [6,7] might be moved into webpage format to encourage higher usage. In addition, a brief evaluation should be carried out after the suggested changes have been implemented on the website.

\section{Conclusions}

Overall, the SkinSupport website is an acceptable and useful resource for people living with skin conditions, and the relatives and friends of individuals with a skin condition, and dermatology professionals. Some of the suggested changes around inaccurate information have already been implemented by BAD. Future decisions on which suggested changes to make will be made by BAD, with the help of this study, once further funding has been secured. After suggested improvements have been made, the website will serve as a valuable resource that can fit into the stepped care model of psychosocial care in dermatology, offering skin-specific self-help to people experiencing distress.

\section{Acknowledgments}

This project was created by the BAD with funding received from the UK Department of Health Voluntary Sector Investment Programme Innovation, Excellence and Strategic Development Fund (award number 251784). 


\section{Conflicts of Interest}

The funders of this project were not involved in the review or approval of the manuscript. Authors NG and MG are employees of the BAD and authors AT and SE were unpaid members of the professional advisory panel for the initial construction of the website. Data were independently collected and analyzed by authors KM and CH under supervision of authors AT and SE.

\section{Multimedia Appendix 1}

Table of qualitative responses (survey and focus group) for participants in the evaluation group. [DOCX File, 25 KB-Multimedia Appendix 1]

\section{References}

1. The All Party Parliamentary Group on Skin. 2013. The Psychological and Social Impact of Skin Diseases on People's Lives URL: https://www.appgs.co.uk/publication/view/

the-psychological-and-social-impact-of-skin-diseases-on-peoples-lives-final-report-2013/ [accessed 2019-02-12]

2. Schofield J, Fleming D, Grindlay D, Williams H. Skin conditions are the commonest new reason people present to general practitioners in England and Wales. Br J Dermatol 2011 Nov;165(5):1044-1050. [doi: 10.1111/j.1365-2133.2011.10464.x] [Medline: 21692764]

3. Grandfield T, Thompson A, Turpin G. An attitudinal study of responses to a range of dermatological conditions using the implicit association test. J Health Psychol 2005 Nov;10(6):821-829. [doi: 10.1177/1359105305057316] [Medline: 16176959]

4. Lavda A, Webb T, Thompson A. A meta-analysis of the effectiveness of psychological interventions for adults with skin conditions. Br J Dermatol 2012 Nov;167(5):970-979. [doi: 10.1111/j.1365-2133.2012.11183.x] [Medline: 22924999]

5. The All Party Parliamentary Group on Skin. 2019. Purpose and Aims URL: http://www.appgs.co.uk/wp-content/uploads/ 2019/02/APPGS 2019 Work Priorities.pdf [accessed 2019-06-10]

6. Thompson A. Self-help for management of psychological distress associated with skin conditions. In: Bewley BA, Taylor RE, Reichenberg JS, Magid M, editors. Practical Psychodermatology. West Sussex: Wiley-Blackwell; 2014:60-65.

7. Beatty L, Lambert S. A systematic review of internet-based self-help therapeutic interventions to improve distress and disease-control among adults with chronic health conditions. Clin Psychol Rev 2013 Jun;33(4):609-622. [doi: 10.1016/j.cpr.2013.03.004] [Medline: 23603521]

8. Bundy C, Pinder B, Bucci S, Reeves D, Griffiths C, Tarrier N. A novel, web-based, psychological intervention for people with psoriasis: the electronic targeted intervention for psoriasis (eTIPs) study. Br J Dermatol 2013 Aug;169(2):329-336. [doi: 10.1111/bjd.12350] [Medline: 23551271]

9. British Association of Dermatologists. URL: http://www.skinsupport.org.uk/ [accessed 2020-04-26]

10. Pasterfield M, Clarke S, Thompson AR. The development of a self-help intervention to build social confidence in people living with visible skin conditions or scars: a think-aloud study. Scars Burn Heal 2019;5:1-8 [FREE Full text] [doi: 10.1177/2059513118822954] [Medline: 30675396]

11. Shah R, Hunt J, Webb TL, Thompson AR. Starting to develop self-help for social anxiety associated with vitiligo: using clinical significance to measure the potential effectiveness of enhanced psychological self-help. Br J Dermatol 2014 Aug;171(2):332-337. [doi: 10.1111/bjd.12990] [Medline: 24665869]

12. Bradbury K, Dennison L, Little P, Yardley L. Using mixed methods to develop and evaluate an online weight management intervention. Br J Health Psychol 2015 Feb;20(1):45-55. [doi: 10.1111/bjhp.12125] [Medline: 25406436]

13. Yardley L, Bishop F. Using mixed methods in health research: benefits and challenges. Br J Health Psychol 2015 Feb;20(1):1-4. [doi: 10.1111/bjhp.12126] [Medline: 25582981]

14. Yardley L, Bishop F. Mixing qualitative and quantitative methods. In: Willig C, Stainton-Rogers W, editors. The Sage Handbook Of Qualitative Research In Psychology. Thousand Oaks, CA: Sage Publications; 2007:352-369.

15. Finlay A, Khan G. Dermatology life quality index (DLQI)-a simple practical measure for routine clinical use. Clin Exp Dermatol 1994 May;19(3):210-216. [doi: 10.1111/j.1365-2230.1994.tb01167.x] [Medline: $\underline{8033378]}$

16. Basra M, Fenech R, Gatt R, Salek M, Finlay A. The dermatology life quality index 1994-2007: a comprehensive review of validation data and clinical results. Br J Dermatol 2008 Nov;159(5):997-1035. [doi: 10.1111/j.1365-2133.2008.08832.x] [Medline: 18795920]

17. Kroenke K, Spitzer R, Williams J. The patient health questionnaire-2: validity of a two-item depression screener. Med Care 2003 Nov;41(11):1284-1292. [doi: 10.1097/01.MLR.0000093487.78664.3C] [Medline: 14583691$]$

18. Kroenke K, Spitzer R, Williams J, Monahan P, Löwe B. Anxiety disorders in primary care: prevalence, impairment, comorbidity, and detection. Ann Intern Med 2007 Mar 6;146(5):317-325. [doi: 10.7326/0003-4819-146-5-200703060-00004] [Medline: 17339617$]$

19. Mayring P. Qualitative content analysis. In: Steinke I, Flick U, Kardoff EV, editors. A Companion to Qualitative Research. Thousand Oaks, CA: Sage Publications; 2004:266-269.

20. Braun V, Clarke V. Using thematic analysis in psychology. Qual Res Psychol 2006 Jan;3(2):77-101 [FREE Full text] [doi: 10.1191/1478088706qp063oa]

21. Glaser B, Strauss A. The Discovery of Grounded Theory. Chicago: Aldine Pub Co; 1967. 


\section{Abbreviations}

BAD: British Association of Dermatologists

DLQI: dermatology quality of life index

GAD: generalized anxiety disorder

GP: general practitioner

PHQ: patient health questionnaire

QCA: qualitative content analysis

Edited by $G$ Eysenbach; submitted 13.11.19; peer-reviewed by B Eapen, D Harcourt; comments to author 10.01.20; revised version received 03.04.20; accepted 08.04.20; published 15.06.20

Please cite as:

Heapy C, Montgomery K, Ersser S, Gass M, Goad N, Thompson AR

A Psychosocial Support Website From the British Association of Dermatologists for People Living With a Skin Condition: Mixed Methods Evaluation

JMIR Dermatol 2020;3(1):e17052

URL: http://derma.jmir.org/2020/1/e17052/

doi: 10.2196/17052

PMID:

(C) Connor Heapy, Kerry Montgomery, Steven Ersser, Matt Gass, Nina Goad, Andrew R Thompson. Originally published in JMIR Dermatology (http://derma.jmir.org), 15.06.2020. This is an open-access article distributed under the terms of the Creative Commons Attribution License (https://creativecommons.org/licenses/by/4.0/), which permits unrestricted use, distribution, and reproduction in any medium, provided the original work, first published in JMIR Dermatology Research, is properly cited. The complete bibliographic information, a link to the original publication on http://derma.jmir.org, as well as this copyright and license information must be included. 